\title{
Choice of structural and technological solutions for engineering preparation of bases for foundations
}

\author{
Dzmitry Klebanyuk ${ }^{1, *}$, Peter Poyta ${ }^{1}$ and Peter Shvedovsky ${ }^{1}$ \\ ${ }^{1}$ Brest State Technical University, 224017, Moskovskaya 267, Brest, Belarus
}

\begin{abstract}
The object of the research is a comprehensive assessment of the choice of structural and technological solutions for the engineering preparation of soil foundations using the methods of dynamic compaction with heavy rammers. It is proposed in the studies to choose the optimal solution according to the criteria of both efficiency and preference with sequential or selective implementation of certain target groups: multipurpose choice from a set of goals; optimization on a set of conditions and in dynamics; multi-vector optimization, etc. Location of the options according to preference, the main directions for optimizing the options for constructive and technological solutions and the best option for the engineering preparation of the base for the foundations were identified on the basis of the proposed method for the experimental site.
\end{abstract}

Key words: foundations, engineering preparation, dynamic compaction, choice of solutions, optimization.

\section{Introduction}

Limited territories with favourable geotechnical conditions for construction necessitate the development of complex, in engineering and geological terms, construction sites.

Local [1-4] and foreign [5-8] practice has shown that the most effective preparation of such areas using methods of dynamic compaction with heavy rammers.

A certain problem when choosing the optimal design solution in terms of structural and technological parameters is created by the need for test compaction of soils at the construction site and multiple uncertainties: initial state of soils; influence of technological parameters on the change in soil properties during the compaction process; peculiarity of taking into account soil compaction in time, etc. [9].

It should also be noted that traditional methods for solving single-criterion problems that implement optimizing factors when restrictions are imposed on all other, other alternative constructive and technological solutions only in terms of economic indicators are not always legitimate, since the cost estimate is most often nonlinear in terms of the optimality of the decision taken [10].

*Corresponding author: klebanyuk.dmitri@gmail.com 
It should also be noted that traditional methods for solving single-criterion problems that implement optimizing factors when restrictions are imposed on all other, other alternative constructive and technological solutions only in terms of economic indicators are not always legitimate, since the cost estimate is most often nonlinear in terms of the optimality of the decision taken [10].

The authors [4] propose to use multivariate models which allow to take into account to a large extent the direct and feedback links of the main factors, their interrelation, as well as the uncertainty of many subjective and objective conditions and parameters of the compaction of soil massifs.

\section{Identification of interdependencies and interrelationships of conditions and factors}

Production experience and most studies [11-14] allow us to note that:

- with an increase in the deformation modulus of soils in the natural state, a decrease in the diameter of the rammers is required, but not less than $1 \mathrm{~m}$, since in this case the soil loosens with the formation of soil lifting zones;

- when compacting soil strata of high thickness, simultaneously with an increase in the diameter of the rammer, it is necessary to increase its mass and discharge height;

- the greatest efficiency of compaction is achieved with optimal moisture content and content of clay particles;

- the more homogeneous the soil the more efficient the compaction with the same energy consumption;

- the increase in the rammer weight often does not lead to a high degree of compaction and uniformity of the compacted base;

- with an increase in the impact energy, the density of the soil increases most significantly at the initial stage of compaction;

- the quality of the compaction is largely determined by the layout and the distance between the compaction points;

- the smaller the diameter of the rammer (at constant impact energy) the smaller the size (radius and depth) of the compaction zone;

- the greater the deformation modulus of natural soils the smaller the tamping diameter should be;

- uniformity of the soil structure in the compaction zone can be achieved by increasing the initial density of dry soil;

- to achieve the required density it is necessary to ram additional volumes of soil into the base at relatively low density of natural soil;

- for soft soils the greater their power, the greater should be the mass of the rammer or the height of its dropping and diameter;

- the greatest depth of the compaction zone while minimizing energy costs can be achieved through the use of rammers of large diameters ( $2 \mathrm{~m}$ or more) at the initial stage of compaction with additional compaction with rammers of a smaller diameter upon reaching failure;

- the greater the thickness of the compacted layer, the distance between the points of compaction of the soil should decrease and at certain values of the thickness of the compacted layer compaction must be performed without the presence of bridges between the indentations, i.e. continuous throughout the site; 
- in the presence of a solid underlying layer within the compacted strata, an increase in the radius of the compacted zone is characteristic, which makes it possible to increase the distance between the points of soil compaction;

- with an increase in the depth of the imprint of the rammer and its diameter, the distance between the sealing points increases proportionally;

- the formation of the soil compaction zone is greatly influenced by the shape of the rammer sole. The use of a rammer with a stepped bottom allows to significantly increase the depth of the compaction zone in comparison with a rammer with a flat bottom;

- the maximum depth of the compaction zone with a sufficiently uniform degree of compaction can be achieved using a two-stage technological process and rammers with a concave spheroid and a convex spherical bottom.

\section{Research method}

Hence, selection of the optimal solutions should be carried out on a group of criteria of efficiency $\left(K_{i j}\right)$ and preference $\left(U_{i}\right)$ (techno-economic, structural,technological and others.), with the need for consistency or selective implementation of certain target groups: multi-purpose selection of a variety of purposes; optimization on multiple conditions; optimization in dynamics, at many stages and many options; multi-vector optimization, etc. $[2,9,10]$.

As parameters of preference, it is advisable to operate with a number of preferences $\left(\bar{X}_{i}\right)$ and vectors of priorities $\left(\lambda_{i}\right)$ and significance $\left(q_{i}\right)$ determined by the method of sequential optimization. Note that the preference series determines the ordered set of local criteria $\bar{x}=x_{1}>x_{2}>\ldots>x_{n}$, the priority vector $\lambda=\left(\lambda_{1}, \lambda_{2}, \ldots, \lambda_{n}\right)$ shows the degree of coincidence of two adjacent performance indicators (PI) in terms of significance and the significance vector - the significance of the $j$ PI in comparison with others [15].

At the same time, performance indicators can be set both precisely (the principle of strict priority) and approximately - by a certain area (the principle of flexible priority).

Using the method of expert evaluations to determine the significance of performance indicators and taking the Bernoulli criterion as the most reliable criterion for the optimality of a constructive and technological solution

$$
K_{i}^{j}=\left\{a_{i} / \max \frac{1}{n} \cdot \sum_{j=1}^{n} \bar{X}_{i j}\right\}, \quad i=1, \bar{m} ; o=1, \bar{n}
$$

where value $\bar{X}_{i j}$ of the $\mathrm{j}$ indicator for the $\mathrm{i}$ option which implements the principle of fair absolute concession and allows one to switch from vector criteria to scalar ones, reducing the multicriteria problem to a single-criterion one.

For significant indicators of the effectiveness of constructive and technological solutions, the following were taken: height of discharge of heavy rammers $(\mathrm{H}, \mathrm{m})$; energy consumption $\left[\sqrt{M \cdot g \cdot H},(m n f \cdot m)^{1 / 2}\right]$; parametric characteristic of compaction $\left(h_{\text {opt }}^{\text {dep }} / h_{\text {dep }}\right)$; index of the degree and uniformity of compaction $\left(\rho_{\text {nat }} / \rho_{\text {req }} \cdot k_{n}\right)$; deformability of the base $\left(E_{m p}, M P a\right)$ and expended work $(N, m n f \cdot m)$. 
The state of the optimal compaction depth is taken as a parametric characteristic of the compaction zone $\left(h_{\text {opt }}^{\text {dep }}\right)$ and final imprint depth $\left(h_{d e p}\right)$, and the index of the degree and uniformity of compaction is natural $\left(\rho_{\text {nat }}\right)$ and required $\left(\rho_{\text {reg }}\right)$ soil density and uniformity coefficient $\left(k_{n}\right)$.

The calculations were carried out in the following stages:

- the initial matrix $(P)$ of the form was determined -

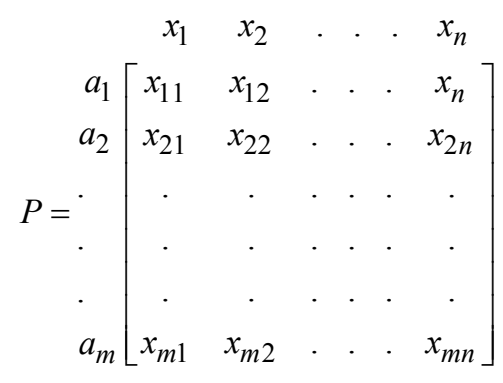

where $a_{1}, \ldots, a_{m}$ - compared options for technical solutions $i=\overline{1, m} ; x_{1}, \ldots, x_{n}$ polydimensional performance indicators $j=\overline{1, n} ; x_{11}, \ldots, x_{m n}$ - values of performance indicators;

- it was normalized $(\bar{P})$ by dependence, which made it possible to compose an efficiency matrix $(F)$ and determine all the sets of agreement $\left(C_{k e}\right)$ and disagreement $\left(H_{k e}\right)$, while the set of agreements consists of indices of those indicators for which the $k$ option is not worse than option $e$ and the set of disagreements consists of indices of the same indicators, according to which $e$ option is superior to $k$ option;

- based on the values of the efficiency indicators $\left(q_{i}\right)$, an efficiency matrix was $\operatorname{compiled}(F=/ \bar{P} / \cdot / q /)$;

- sets of agreement and disagreement were determined for each pair of options;

- indices of agreement $\left(C_{k e}\right)$ and disagreement $\left(d_{k e}\right)$ were determined, reflecting the preference of one option over another, from which the corresponding matrices of agreement $(C)$ and disagreement $(D)$ are formed;

- the dominant agreement $\left(D_{c}\right)$ and disagreement $\left(D_{H}\right)$ matrices were constructed based on the correspondences of the relative weightings of the indicators $l_{k e}$ and $d_{k e}$ :

$$
l_{k e}=\left\{\begin{array}{c}
1, \text { if } C_{k e} \geq \bar{C} \\
0, \text { if } C_{k e}<\bar{C}
\end{array} ; \quad d_{k e}=\left\{\begin{array}{l}
1, \text { if } d_{k e} \geq \bar{d} \\
0, \text { if } d_{k e}<\bar{d}
\end{array},\right.\right.
$$

where $C$ is the average index of agreement, and $\bar{d}$ is the average index of disagreement.

As the significance of performance indicators are taken according to [6] -

$$
q_{1}=0,193 ; q_{2}=0,196 ; q_{3}=0,229 ; q_{4}=0,202 ; q_{5}=0,180 \text {. }
$$




\section{Results and discussion}

The initial matrix was formed for an experimental construction site in the geological structure of which technogenic formations are involved, represented by dumps of soil from sands of various sizes, mixed with sandy loam with inclusions (up to $3 \%$ ) of gravel. The dumping time is more than 10 years, which determines their sufficiently compacted state. The thickness of the deposits is from 0.5 to $0.7 \mathrm{~m}$. The bedrock deposits are represented by fine and medium sands of low strength and medium strength. The thickness of the sandy strata is from 2.0 to $5.0 \mathrm{~m}$. Loams occur from a depth of 7.0-8.0 m.

Groundwater - free-flow with a steady level in the range of 4.6-5.1 m.Water-bearing soils - fine and medium sands with the following averaged physical and mechanical characteristics:

Based on the production capabilities of the general contractor, the following indicators of the effectiveness of constructive and technological solutions were adopted (Table 1).

Table 1. Significant indicators of the effectiveness of constructive and technological solutions for soil compaction with heavy rammers.

\begin{tabular}{|c|c|c|c|c|c|c|c|}
\hline \multirow[b]{2}{*}{ 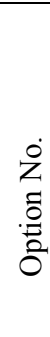 } & \multirow{2}{*}{\multicolumn{2}{|c|}{$\begin{array}{l}\text { Constructive and } \\
\text { technological } \\
\text { solutions }\end{array}$}} & \multicolumn{5}{|c|}{ Efficiency criteria } \\
\hline & & & 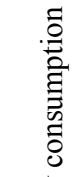 & $\begin{array}{l}\text { Parametric } \\
\text { characteristic } \\
\text { of the }\end{array}$ & $\begin{array}{l}\text { Index of } \\
\text { the degree } \\
\text { and } \\
\text { uniformity }\end{array}$ & $\begin{array}{c}\text { Deformability } \\
\text { of the base }\end{array}$ & $\begin{array}{c}\text { Work } \\
\text { expended }\end{array}$ \\
\hline 1 & \multirow{2}{*}{$\begin{array}{c}\text { Drop } \\
\text { height, } \mathrm{m}\end{array}$} & $H_{1}=8$ & 20,0 & 4,5 & 0,82 & 16,8 & 12,3 \\
\hline 2 & & $H_{2}=10$ & 23,2 & 4,7 & 0,86 & 19,3 & 15,6 \\
\hline 3 & \multirow{2}{*}{$\begin{array}{c}\text { Distance } \\
\text { between } \\
\text { compaction } \\
\text { points, m }\end{array}$} & $l_{1}=4,0$ & 21,3 & 4,6 & 0,84 & 18,4 & 10,4 \\
\hline 4 & & $l_{2}=6,5$ & 20,0 & 4,5 & 0,82 & 20,2 & 11,9 \\
\hline 5 & \multirow{2}{*}{$\begin{array}{l}\text { Ramming } \\
\text { weight, } t\end{array}$} & $M_{1}=5,0$ & 20,8 & 4,58 & 0,79 & 20,3 & 13,5 \\
\hline 6 & & $M_{2}=7,0$ & 19,6 & 4,62 & 0,82 & 21,2 & 15,2 \\
\hline
\end{tabular}

Note: The variant with the index "1" is accepted as the basic one.

According to the average index of agreement $\bar{C}=0,513$ and disagreement index $\bar{d}=0,476$ dominant matrices of agreement $D_{c}$ and disagreement $D_{n}$, aggregated matrix the matrix of dominance $D_{a}=D_{c} \cdot D_{n}$ was determined and in accordance with the criteria of agreement and disagreement the non-dominant options are the first, fourth and sixth options.

No less important is the question of ordering options according to their preference. We have used the most acceptable method of ordering which is suitable for a set of cardinal (numerical) and ordinal indicators of performance of the compared options.

As an estimate of the ordering of the variants $\beta_{q}(q=1,2, \ldots, m !)$ the following dependence was taken: 


$$
\beta_{q}=\sum_{k, e=1}^{m} \cdot \sum_{\ni C_{k e}} q_{j}-\sum_{k, e=1}^{m} \cdot \sum_{\ni H_{k e}} q_{j}, q=1,2, \ldots, m !
$$

where

$$
\left.\begin{array}{l}
C_{k e}=\left\{j / X_{k j}>X_{e j}\right\}, k, e=1, \bar{m} ; k \neq e ; \\
H_{k e}=\left\{j / X_{k j}>X_{e j}\right\}, k, e=1, \bar{m} ; k \neq e
\end{array}\right\}
$$

and then the agreed (the best) ordering will be mo, for which the value $\beta \mathrm{q}$ is the largest. It should be borne in mind that the most consistent ordering is identical to the series of preferable options.

Significant cardinal and ordinal indicators are shown in Table 2.

\begin{tabular}{|c|c|c|c|c|c|c|}
\hline \multirow{2}{*}{ Indicator of efficiency } & \multirow{2}{*}{$\begin{array}{l}\text { Indicator } \\
\text { index }\end{array}$} & \multicolumn{4}{|c|}{ Options } & \multirow{2}{*}{$\begin{array}{l}\text { Significanc } \\
\text { e of the } \\
\text { indicator }\end{array}$} \\
\hline & & №1 & №2(3) & №3(5) & №4(6) & \\
\hline 1 & 2 & 3 & 4 & 5 & 6 & 7 \\
\hline Work expended & $\mathrm{X}_{1}$ & 12,3 & 10,4 & 13,5 & 15,2 & 0,15 \\
\hline $\begin{array}{l}\text { Compaction uniformity } \\
\text { index }\end{array}$ & $\mathrm{X}_{2}$ & 0,84 & 0,85 & 0,81 & 0,83 & 0,11 \\
\hline Deformability of the base & $X_{3}$ & 34,2 & 39,7 & 34,7 & 36,9 & 0,18 \\
\hline Energy consumption & $\mathrm{X}_{4}$ & 20,0 & 21,3 & 20,8 & 19,6 & 0,16 \\
\hline $\begin{array}{l}\text { Parametric characteristic } \\
\text { of the compaction zone }\end{array}$ & $\mathrm{X}_{5}$ & 4,5 & 4,6 & 4,58 & 4,62 & 0,09 \\
\hline $\begin{array}{l}\text { Degree of homogeneity of } \\
\text { the compacted base }\end{array}$ & $\mathrm{X}_{6}$ & III & III & II & II & 0,06 \\
\hline Relative energy costs & $X_{7}$ & II & III & II & II & 0,07 \\
\hline Operational reliability & $\mathrm{X}_{8}$ & III & III & II & III & 0,05 \\
\hline $\begin{array}{l}\text { Degree of dynamic impact } \\
\text { on existing adjacent } \\
\text { objects }\end{array}$ & $\mathrm{X}_{9}$ & III & III & III & II & 0,03 \\
\hline $\begin{array}{l}\text { Internal factors beyond the } \\
\text { control of the designers }\end{array}$ & $\mathrm{X}_{10}$ & II & III & III & II & 0,02 \\
\hline $\begin{array}{l}\text { Internal factors beyond the } \\
\text { control of the contractor }\end{array}$ & $\mathrm{X}_{11}$ & III & I & II & III & 0,02 \\
\hline $\begin{array}{l}\text { External factors of the } \\
\text { features of the compaction } \\
\text { devices }\end{array}$ & $\mathrm{X}_{12}$ & I & III & III & III & 0,01 \\
\hline $\begin{array}{l}\text { External factors of } \\
\text { qualification of work } \\
\text { performers }\end{array}$ & $\mathrm{X}_{13}$ & II & II & III & III & 0,02 \\
\hline $\begin{array}{l}\text { Factors of randomness and } \\
\text { uncertainty }\end{array}$ & $\mathrm{X}_{14}$ & I & II & I & II & 0,05 \\
\hline
\end{tabular}

Table 2. Cardinal and ordinal indicators of the effectiveness of structural and technological solutions for compaction of bases with heavy rammers.

Note. Ordinal indicators I, II and III for levels and conditions characterize respectively "high-medium-low", and for needs - "small-medium-large"

The preference of the options was analyzed on the basis of entropy $[16,17]$ for which transforming the initial matrix $(P)$ into the calculated one $(\bar{P})$ the levels of entropy $\left(E_{j}\right)$, 
levels of variability $\left(d_{j}\right)$ and the absolute weightings of indicators $\left(q_{j}\right)$ were determined according to the dependences -

$$
\left.\begin{array}{l}
\bar{P}_{i j}=X_{i j} / \sum_{i=1}^{m} X_{i j} ; \quad E_{j}=k \cdot \sum_{i=1}^{m} p_{i j} \ln p_{i j}, j=1, \bar{n} \\
d_{j}=1-E_{j}, j=1, \bar{n} ; \quad q_{j}=d_{j}:\left(\sum_{j=1}^{n} d_{j}\right), j=1, \bar{n} .
\end{array}\right\}
$$

The obtained criteria for the weighted average success of the decision made make it possible to arrange the options according to preference as follows: $a_{4}>a_{6}>a_{1}$, i.e. $a_{4}$ is "no worse than $a_{6}$ ", etc.

No less important is the issue of directed improvement of the selected (out of all competitive) options for constructive and technological solutions, which is based on a common utility function that takes into account both independence in preference and independence in utility [18-21].

The verification of the fulfillment of the conditions of independence in terms of preference and utility, carried out for all pairs of variables, made it possible to obtain equivalent pairs of multidimensional alternatives, and given that the general utility function should have a multiplicative form

$$
u\left(x_{i}\right)=\frac{1}{k} \prod_{i=1,4} 1+k_{i} \cdot k \cdot u_{i}\left(x_{i}\right)-1 ; \quad \sum_{i=1}^{4} k_{i} \neq 1,
$$

showed that the best constructive and technological option is the a 4 option.

\section{Conclusions}

1. The conducted studies allow us to note that an arbitrary choice of design parameters of heavy rammers and not taking into account the technological features and patterns of soil compaction dynamics leads to an increase in the cost of engineering preparation of a construction site and does not allow achieving the required degree and uniformity of soil compaction at relatively acceptable energy costs.

2. Optimization of the size and shape of the rammers and accordingly the mass, dropping height, the distance between the compaction points, the technology and organization of work requires a complete and reliable account of both engineering and geological conditions as well as design and technological parameters and their attendant factors.

3. Since the indicators of the efficiency of the design option are a large group of possible alternatives, when choosing the optimal resource-saving option, it is advisable to use the scheme we proposed, which allows the main tasks - drawing up a series of preferences and choosing a design option - to be solved at any level using the capabilities of computer-aided design (CAD).

\section{References}

1. D.N. Klebanyuk, P. S. Poita A, Vestnik BSTU 1, pp. 77-81 (2013)

2. P. V. Shvedovsky, D. N. Klebanyuk, P. S. Poyta, Sb. articles II MNTK "Theory and practice of research and design in construction with the use of CAD", pp. 50-58 (2018) 
3. P.S. Poita, P.V.Shvedovskiy, D.N. Klebanyuk, Vestnik BSTU 1, pp. $72-77$ (2003)

4. D. N. Klebanyuk, P. S. Poyta, P. V. Shvedovsky, Geotechnics of Belarus: science and practice: materials of the Intern. scientific and technical conferences 2, pp. 109-120 (2013)

5. L. M'enard, Y. Broise, G'eotechnique 25(1), pp. 3-18 (1975)

6. A. Oshima, N. Takada, International Conference on Soil Mechanics \& Foundation Engineering 13(4), pp 1641-1194 (1994)

7. J. Pan, Z.M. Hwang, Sino Geotech 51, pp. 35-50 (1995)

8. A. Perucho, C. Olalla, Ground Improvement 10(2), pp. 55-68 (2006)

9. P.S. Poyta, Vestnik BSTU 1, pp. 109-110 (2003)

10. E.K. Zavadskas, Complex assessment and selection of resource-saving solutions in construction (Vilnius, Mokslas, 1987)

11. V.B. Shvets, Compaction of soil bases with heavy rammers (Rosstroyizdat, 1988)

12. Y.K. Chow, D.M. Yong, K.Y., Yong, S.L. Lee, Journal of Geotechnical Engineering 118(8), pp. 1141-1157 (1992)

13. H.Y. Fang, G.W. Ellis, Frity Engineering Laboratory Report 462-06 (Lehigh University, Bethlehem, 1983)

14. S. Helwany, Applied Soil Mechanics ABAQUS applications (New York, 2007)

15. B.G. Mirkin, Solution of group choice problems (Moscow, Nauka, 1998)

16. G.K. Bondarik, Fundamentals of the theory of variability of engineering-geological properties of rocks (Moscow, Nedra, 1971)

17. V.B. Mikheev, I.V. Shitova, Foundations, foundations and underground structures 71, pp. 16-21 (1980)

18. M. Smits, J.H. Th, L. De Quelerij, Proceedings, 12th international Conference of Soil Mechanics and Fundations Enginering 2, pp. 1419-1422 (1989)

19. P.W. Mayne, J.S.Jones, J.C. Dumas, Journal Geotech Eng, ASCE 110(JT6), pp. 757774 (1984)

20. C.J. Poran, K.S. Heh, J.A. Rodriguez, Soils and Foundations 32(4), pp 81-92 (1992)

21. A. Scott, W. Pearce, G'eotechnique 25(1), pp. 19-30 (1975) 